\title{
(E)-Selective Horner-Wadsworth-Emmons reaction of aldehydes with bis-(2,2,2-trifluoroethyl)phosphonoacetic acid
}

\author{
Shigeki Sano,* Yuka Takemoto, and Yoshimitsu Nagao* \\ Faculty of Pharmaceutical Sciences, The University of Tokushima, Sho-machi, \\ Tokushima 770-8505, Japan \\ E-mail: ssano@ph2.tokushima-u.ac.jp
}

Dedicated to Professor Keiichiro Fukumoto on his $\mathbf{7 0}^{\text {th }}$ birthday

(received 29 May 03; accepted 30 June 03; published on the web 14 July 03)

\begin{abstract}
The stereoselective Horner-Wadsworth-Emmons reaction of aldehydes with bis-(2,2,2trifluoroethyl)phosphonoacetic acid utilizing $i$-PrMgBr afforded $(E)-\alpha, \beta$-unsaturated carboxylic acids as the major products. bis-(2,2,2-Trifluoroethyl)phosphonoacetic acid was prepared by enzymatic hydrolysis of the corresponding methyl ester with porcine liver esterase.
\end{abstract}

Keywords: Horner-Wadsworth-Emmons reaction, olefination, magnesium, $\alpha, \beta$-unsaturated carboxylic acid, $\alpha, \beta$-unsaturated ester

\section{Introduction}

One of the most powerful methods for the stereoselective preparation of $\alpha, \beta$-unsaturated esters is the Horner-Wadsworth-Emmons (HWE) reaction of aldehydes. ${ }^{1}$ The HWE reaction of aldehydes with methyl bis-(2,2,2-trifluoroethyl)phosphonoacetate (1) is a convenient method for the $Z$-selective synthesis of $\alpha, \beta$-unsaturated esters. ${ }^{2}$ It is also known that ethyl 2-fluoro-2diethylphosphonoacetate (2) is an efficient HWE reagent for bringing about $E$-selective fluoroolefination. ${ }^{3}$ According to the Cahn-Ingold-Prelog (CIP) convention, $(Z)$ - $\alpha, \beta$-unsaturated esters from phosphonoacetate $\mathbf{1}$, and $(E)$ - $\alpha$-fluoro- $\alpha, \beta$-unsaturated esters from phosphonoacetate 2 have the same geometric arrangement about their double bonds. Recently, we have reported the Mg(II)-promoted Z-selective HWE reaction of aldehydes with 2-fluoro-2diethylphosphonoacetic acid (3) for the stereoselective synthesis of (Z)- $\alpha$-fluoro- $\alpha, \beta$-unsaturated esters. ${ }^{4}$ That is to say, the $Z$-selectivity of the resulting $\alpha$-fluoro- $\alpha, \beta$-unsaturated carboxylic acids was achieved by utilizing phosphonoacetic acid 3 and $i-\mathrm{PrMgBr}$ in the $\mathrm{HWE}$ reaction of various aldehydes in THF under reflux conditions. In an effort to understand the origin of the stereoselectivities in the HWE reaction, we investigated the HWE reaction of bis-(2,2,2- 
trifluoroethyl)phosphonoacetic acid (4) with various aldehydes 7a-d utilizing i-PrMgBr. We now report on phosphonoacetic acid $\mathbf{4}$ with an $E$-selective HWE reagent for the preparation of $\alpha, \beta$-unsaturated esters.

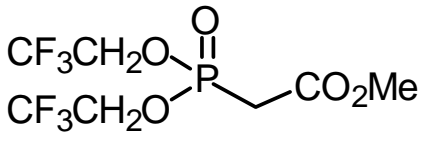

1<smiles>CCOC(=O)C(F)P(=O)(OCC)OCC</smiles>

2<smiles>CCOP(=O)(OCC)C(F)C(=O)O</smiles>

3

\section{Figure 1}

\section{Results and Discussion}

The HWE reagent, bis-(2,2,2-trifluoroethyl)phosphonoacetic acid (4) was prepared by enzymatic hydrolysis of methyl bis-(2,2,2-trifluoroethyl)phosphonoacetate (1) with porcine liver esterase (PLE, Sigma; E-2884) in 92\% yield, as shown in Scheme 1. Attempts to hydrolyze the phosphonoacetate $\mathbf{1}$ under aqueous alkaline conditions were unsuccessful, even though methyl methyl-(2,2,2-trifluoroethyl)phosphonoacetate (5) and methyl dimethylphosphonoacetate (6) were obtained in 51 and 20\% yields, respectively. Ghosh et al. reported a non-enzymatic procedure for the synthesis of phosphonoacetic acid 4 from 2,2,2-trifluoroethanol, methylphosphonic dichloride, and benzyl chloroformate. ${ }^{5}$
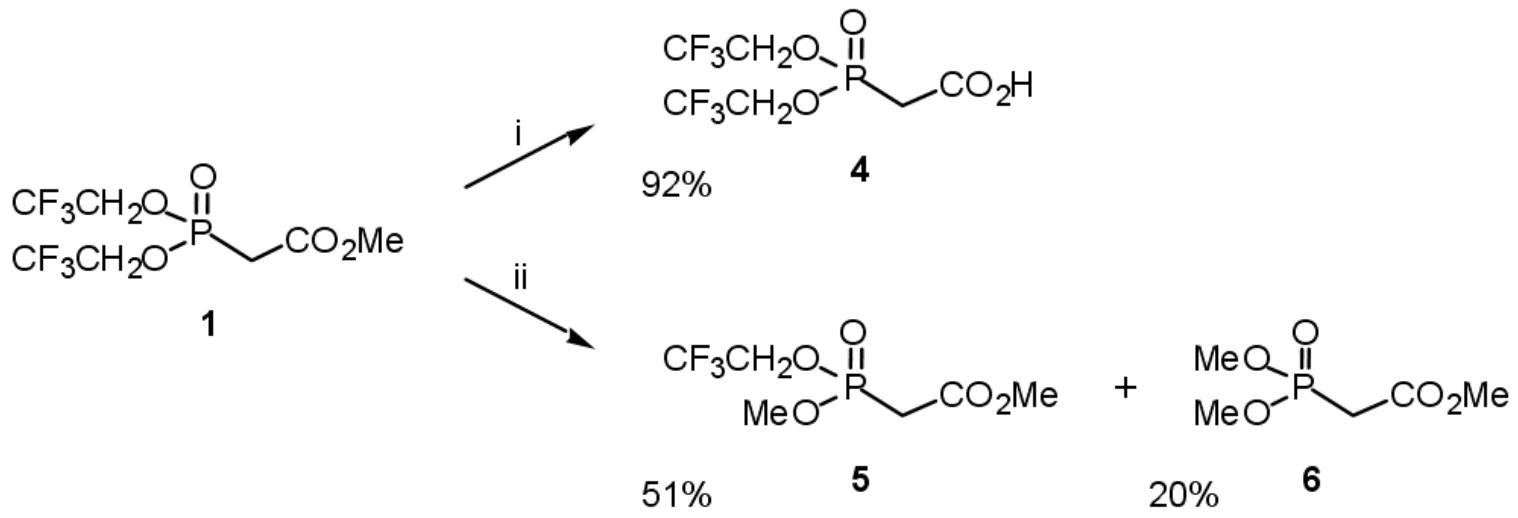

(i) PLE / 0.1 Mphosphate buffer ( $\mathrm{pH} 7.4$ )-acetone (9:1) / rt / 2h -

(ii) $1 \mathrm{MNaOH}-\mathrm{MeOH}(1: 1) / 0{ }^{\circ} \mathrm{C} / 45 \mathrm{~min}$.

\section{Scheme 1}


The HWE reaction of the substituted phosphonoacetic acid $\mathbf{4}$ with the aldehydes $\mathbf{7 a - d}$ in the presence of $i$-PrMgBr afforded the $\alpha, \beta$-unsaturated carboxylic acids 8a-d. Esterification of 8ad with an excess amount of trimethylsilyldiazomethane $\left(\mathrm{TMSCHN}_{2}\right)^{6}$ provided the desired $\alpha, \beta$ unsaturated esters 9a-d, without the isolation of $\mathbf{8 a}-\mathbf{d}$, as shown in Scheme 2. All the results of the HWE reactions employing the phosphonoacetic acid 4 are summarized in the following Tables 1 and 2. Under reflux conditions in THF, utilizing $i$-PrMgBr, the reaction of the phosphonoacetic acid 4 with 3-phenylpropionaldehyde (7a) proceeded in a E-selective manner $\left(E: Z=87: 13\right.$ ), while under $0{ }^{\circ} \mathrm{C}$ conditions, the $E: Z$ ratio was $77: 23$ (Table 1 , entries 3 and 4 ). A temperature-dependent improvement of E-selectivity up to 95:5 (E:Z) was found in the reaction of 4 with 7a under reflux conditions in toluene (Table 1, entry 1). The HWE reactions of 4 with 7a using $n$-BuLi resulted in low yields (18-52\%) (Table 1, entries 5-7). In the HWE reactions of the phosphonoacetate 1 with 7a employing $i$-PrMgBr $(1.25 \mathrm{~mol}$ eq) under reflux conditions in toluene, the $E: Z$ stereoselectivity of the products 9 a was moderate, with a ratio of $78: 22$, in $77 \%$ yield.

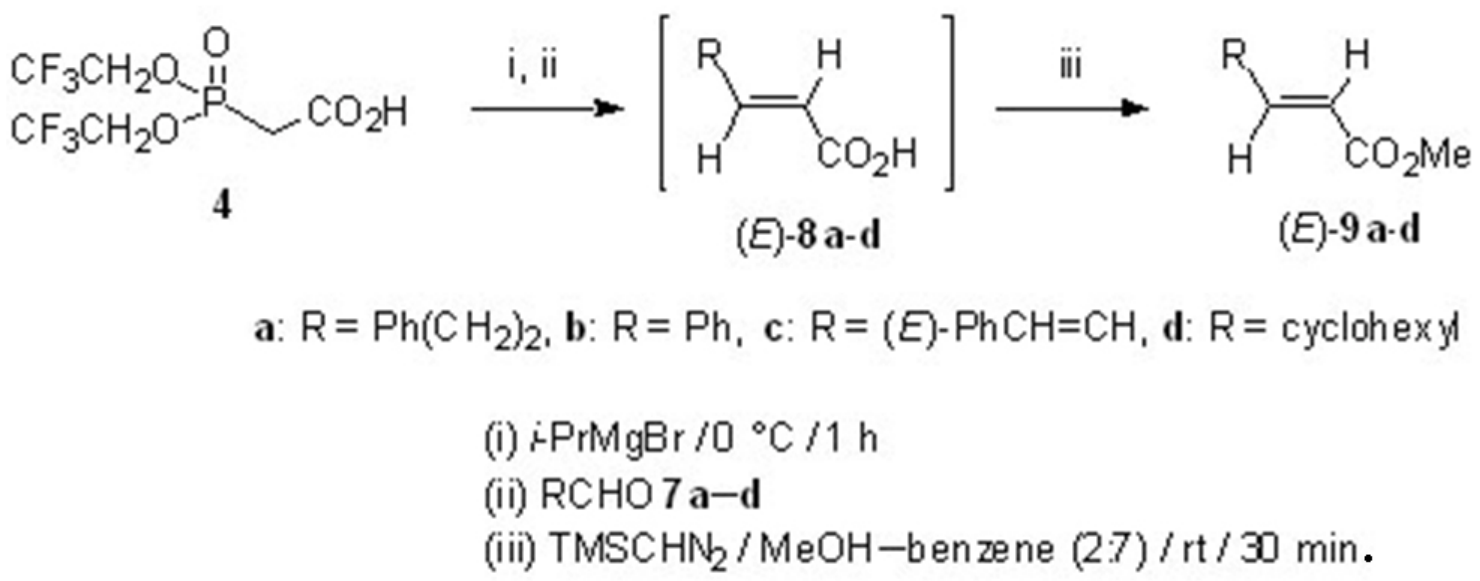

\section{Scheme 2}

In the HWE reaction of the phosphonoacetic acid $\mathbf{4}$ with aldehydes $\mathbf{7 b}-\mathbf{d}, \alpha, \beta$-unsaturated carboxylic acids $\mathbf{8 b}-\mathbf{d}$ were obtained in a fairly good diastereomer ratio up to $>99:<1(E: Z)$, as shown in Table 2. A lowering of the reaction temperature did not result in lowering of the stereoselectivity in the HWE reactions of $\mathbf{4}$ with benzaldehyde (7b), and the $\alpha, \beta$-unsaturated ester (E)-9b was obtained as a sole product in each reaction (Table 2, entries 1-3). The geometry and the diastereomer ratios of $\mathbf{9 a}-\mathbf{d}$ were confirmed on the basis of the coupling constants between the olefinic protons, and integration of the appropriate proton absorptions determined by ${ }^{1} \mathrm{H} \mathrm{NMR}\left(400 \mathrm{MHz}, \mathrm{CDCl}_{3}\right)$ analysis. 
Table 1. Horner-Wadsworth-Emmons reactions of phosphonoacetic acid 4 with aldehyde $\mathbf{7 a}$

\begin{tabular}{|c|c|c|c|c|c|c|}
\hline Entry & Base & Solvent & Temperature & $\begin{array}{l}\text { Time } \\
\text { (h) }\end{array}$ & $\begin{array}{l}\text { Yield of } \\
9 \mathbf{a}(\%)^{\mathrm{b}}\end{array}$ & $E-8 \mathbf{a}: Z-8 \mathbf{a}^{c}$ \\
\hline 1 & $i$-PrMgBr & toluene & reflux & 1 & 82 & $95: 5$ \\
\hline 2 & $i-\mathrm{PrMgBr}$ & dioxane & reflux & 1 & 93 & $90: 10$ \\
\hline 3 & $i$-PrMgBr & THF & reflux & 1 & 96 & $87: 13$ \\
\hline 4 & $i$-PrMgBr & THF & $0{ }^{\circ} \mathrm{C}$ & 2.5 & 74 & $77: 23$ \\
\hline 5 & $n$-BuLi & toluene & reflux & 1 & 18 & $91: 9$ \\
\hline 6 & $n$-BuLi & THF & reflux & 1 & 33 & $42: 58$ \\
\hline 7 & $n$-BuLi & THF & $0^{\circ} \mathrm{C}$ & 17 & 52 & $41: 59$ \\
\hline
\end{tabular}

Table 2. $\mathrm{Mg}(\mathrm{II})$-promoted Horner-Wadsworth-Emmons reactions of phosphonoacetic acid 4 with aldehydes $\mathbf{7 b}-\mathbf{d}^{\mathrm{a}}$

\begin{tabular}{ccccccc}
\hline Entry & Aldehyde & Solvent & Temperature & $\begin{array}{c}\text { Time } \\
(\mathrm{h})\end{array}$ & $\begin{array}{c}\text { Yield } \\
(\%)\end{array}$ & $E: Z^{\mathrm{c}}$ \\
\hline $\mathbf{1}$ & $\mathbf{7 b}$ & toluene & reflux & 1 & $61(\mathbf{9 b})$ & $>99:<1(\mathbf{8 b})$ \\
$\mathbf{2}$ & $\mathbf{7 b}$ & THF & reflux & 1 & $95(\mathbf{9 b})$ & $>99:<1(\mathbf{8 b})$ \\
$\mathbf{3}$ & $\mathbf{7 b}$ & THF & $0^{\circ} \mathrm{C}$ & 4 & $90(\mathbf{9 b})$ & $>99:<1(\mathbf{8 b})$ \\
$\mathbf{4}$ & $\mathbf{7 c}$ & toluene & reflux & 1 & $58(\mathbf{9 c})$ & $96: 4(\mathbf{8 c})$ \\
$\mathbf{5}$ & $\mathbf{7 c}$ & THF & reflux & 1 & $88(\mathbf{9 c})$ & $95: 5(\mathbf{8 c})$ \\
$\mathbf{6}$ & $\mathbf{7 d}$ & toluene & reflux & 1 & $72(\mathbf{9 d})$ & $97: 3(\mathbf{8 d})$ \\
$\mathbf{7}$ & $\mathbf{7 d}$ & THF & reflux & 1 & $93(\mathbf{9 d})$ & $93: 7(\mathbf{8 d})$ \\
\hline
\end{tabular}

${ }^{\mathrm{a}} 4$ / i-PrMgBr / 7 (1.2:2.5:1 molar ratio). ${ }^{\mathrm{b}}$ Isolated yields. ${ }^{\mathrm{c}}$ Determined by ${ }^{1} \mathrm{H}$ NMR (400 MHz, $\left.\mathrm{CDCl}_{3}\right)$ analysis.

A plausible mechanism for the HWE reactions of phosphonoacetic acid $\mathbf{4}$ with aldehyde $\mathbf{7}$ is shown in Scheme 3. On the basis of the experimental results described above, the stereoselective outcome with $E$-selectivity in the $\mathrm{Mg}(\mathrm{II})$-promoted HWE reactions of 4 with 7 can be rationalized in terms of thermodynamic control in a manner similar to that proposed by us in the Mg(II)-promoted HWE reactions of phosphonoacetic acid 3 with 7. ${ }^{4 a, 7}$ The step involving the nucleophilic addition of dianion $\mathbf{1 0}$ to $\mathbf{7}$ toward the pro-(E)- or pro-(Z)-oxyanion must be effectively reversible because of the oxophilicity of $\mathrm{Mg}(\mathrm{II}){ }^{8} \quad$ Also, a repulsive interaction between the carboxylate anion of $\mathbf{1 0}$ and $\mathrm{R}$ of $\mathbf{7}$ may be unfavorable for occupying a pro-(Z)transition state. Consequently, the (E)- $\alpha, \beta$-unsaturated carboxylic acid $(E)-\mathbf{8}$ was obtained as the thermodynamically controlled major product. 


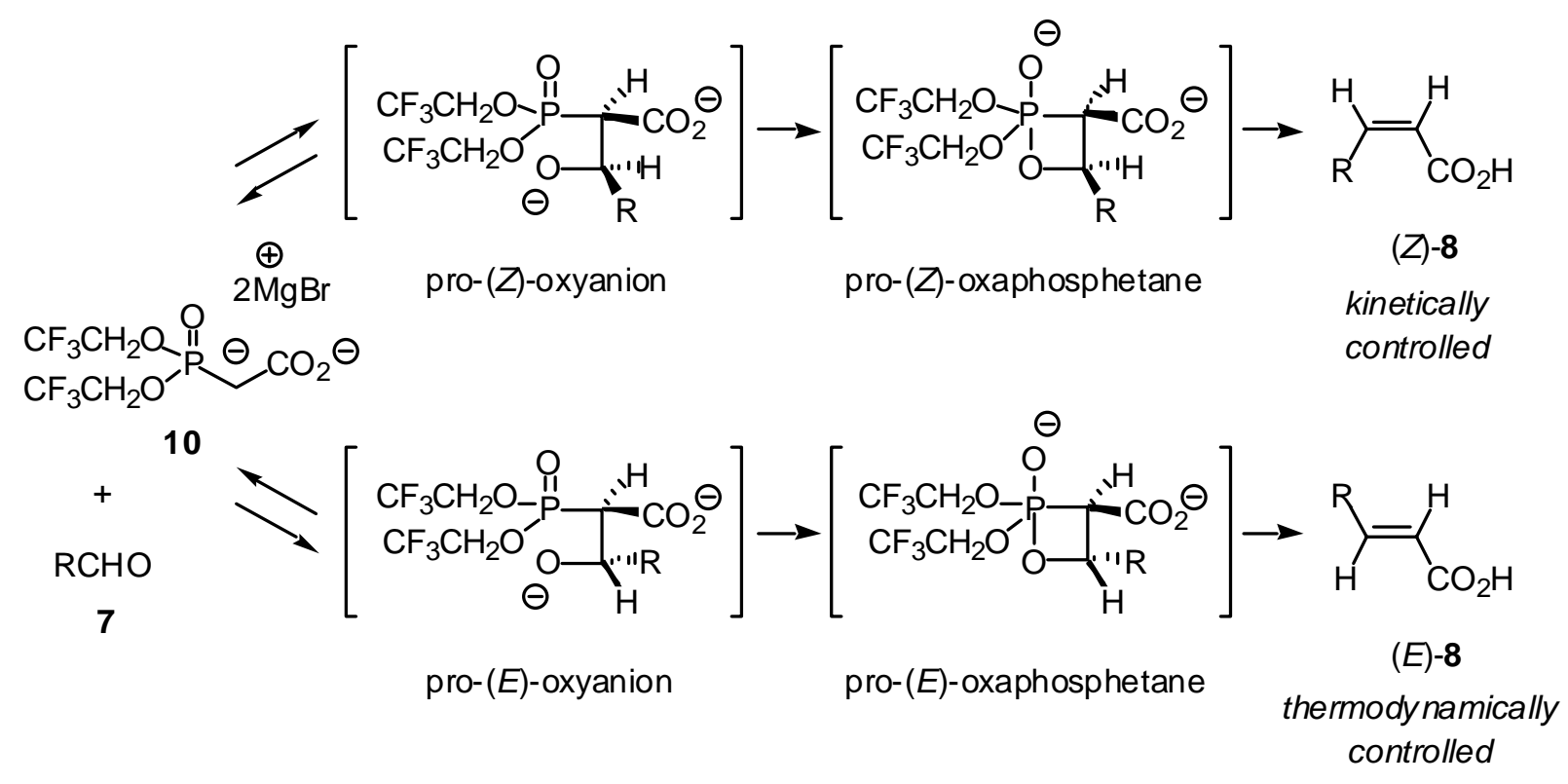

Scheme 3

\section{Conclusions}

We were able to demonstrate that $(E)$ - $\alpha, \beta$-unsaturated esters 9a-d were obtained by the HWE reaction of aldehydes $\mathbf{7 a - d}$ with bis-(2,2,2-trifluoroethyl)phosphonoacetic acid (4), readily prepared by the enzymatic hydrolysis of methyl bis-(2,2,2-trifluoroethyl)phosphonoacetate (1). In light of the well-known fact that the phosphonoacetate $\mathbf{1}$ is a powerful HWE reagent for the preparation of $(Z)$ - $\alpha, \beta$-unsaturated esters, the E-selective HWE reaction of aldehydes $7 \mathbf{a}-\mathbf{d}$ with the substituted phosphonoacetic acid $\mathbf{4}$ provides significant information on the mechanisms of the HWE reaction.

\section{Experimental Section}

General Procedures. All melting points were determined on a Yanagimoto micro melting point apparatus and are uncorrected. IR spectra were obtained using a JASCO FT/IR-420 IR Fourier transform spectrometer. ${ }^{1} \mathrm{H}-\mathrm{NMR}(400 \mathrm{MHz})$ and ${ }^{13} \mathrm{C}-\mathrm{NMR}(75 \mathrm{MHz})$ spectra were recorded on JEOL JNM-AL400 and JEOL JNM-AL300 spectrometers, respectively. Chemical shifts are given in $\delta$ values (ppm) using tetramethylsilane (TMS) as an internal standard. Electron impact (EI)- MS were recorded on a JEOL JMS SX-102A spectrometer. Elemental combustion analyses were performed using a Yanagimoto CHN CORDER MT-5. All reactions were monitored by TLC employing 0.25-mm silica gel plates (Merck 5715; $60 \mathrm{~F}_{254}$ ). Preparative TLC (PTLC) was 
performed on 0.5-mm silica gel plates (Merck 5744; $60 \mathrm{~F}_{254}$ ). Column chromatography was carried out on silica gel [Nacalai Tesque 75SL-II-PREP; 70-300 mesh, Kanto Chemical N60 (spherical, neutral); 63-210 $\mu \mathrm{m}$ ]. “The usual workup” refers to washing an organic portion with brine, drying over anhydrous $\mathrm{MgSO}_{4}$, filtration, and concentration in vacuo. Anhydrous THF, 1,4-dioxane, and toluene were used as purchased from Kanto Chemical. All aldehydes were distilled prior to use. All other reagents were used as purchased.

Bis-(2,2,2-trifluoroethyl)phosphonoacetic acid (4). PLE (Sigma; E-2884, 800 units/mmol) was added to a stirred solution of methyl bis-(2,2,2-trifluoroethyl)phosphonoacetate (1) (228 $\mu \mathrm{L}$, $1.08 \mathrm{mmol}$ ) in $0.1 \mathrm{M}$ phosphate buffer (pH 7.4)-acetone $(9: 1)$ (30 mL) at room temperature. After being stirred at room temperature for $2 \mathrm{~h}$, the reaction mixture was treated with $10 \% \mathrm{HCl}$ and then extracted with AcOEt (70 mL x5). The extract was subjected to the usual workup to give an oily residue, which was purified by silica gel (Nacalai Tesque 75SL-II-PREP) column chromatography [ $\left.\mathrm{CHCl}_{3} / \mathrm{MeOH}(9: 1)\right]$ to afford 4 (303 mg, 92\%) as colorless plates, mp 44.5$46.5{ }^{\circ} \mathrm{C}\left(\mathrm{Et}_{2} \mathrm{O}-n\right.$-hexane); ${ }^{1} \mathrm{H}$ NMR $\left(400 \mathrm{MHz}, \mathrm{CDCl}_{3}\right) \delta 3.22\left(2 \mathrm{H}, \mathrm{d},{ }^{2} J_{\mathrm{H}, \mathrm{P}}=21.2 \mathrm{~Hz}\right), 4.40-$ $4.60(4 \mathrm{H}, \mathrm{m}), 5.45(1 \mathrm{H}, \mathrm{bs}) ;{ }^{13} \mathrm{C} \mathrm{NMR}\left(75 \mathrm{MHz}, \mathrm{CDCl}_{3}\right) \delta 33.7\left(\mathrm{~d},{ }^{1} \mathrm{~J}_{\mathrm{C}, \mathrm{P}}=144.5 \mathrm{~Hz}\right.$ ), 63.0 (quart $\mathrm{d},{ }^{2} J_{\mathrm{C}, \mathrm{F}}=38.6 \mathrm{~Hz},{ }^{2} J_{\mathrm{C}, \mathrm{P}}=5.6 \mathrm{~Hz}$ ), 122.4 (quart d, $\left.{ }^{1} J_{\mathrm{C}, \mathrm{F}}=277.2 \mathrm{~Hz},{ }^{3} J_{\mathrm{C}, \mathrm{P}}=8.1 \mathrm{~Hz}\right), 167.0\left(\mathrm{~d},{ }^{2} J_{\mathrm{C}, \mathrm{P}}\right.$ $=4.4 \mathrm{~Hz})$; IR $(\mathrm{KBr}) 2981,1730,1295,1184,1075 \mathrm{~cm}^{-1}$; EI-MS calcd for $\mathrm{C}_{6} \mathrm{H}_{7} \mathrm{O}_{5} \mathrm{~F}_{6} \mathrm{P} \mathrm{MW}$ 303.9935, found $\mathrm{m} / \mathrm{e} 303.9936\left(\mathrm{M}^{+}\right)$; Anal. calcd for $\mathrm{C}_{6} \mathrm{H}_{7} \mathrm{O}_{5} \mathrm{~F}_{6} \mathrm{P}: \mathrm{C}, 23.70$; $\mathrm{H}, 2.32$. Found: C, 23.75; H, 2.32\%.

Methyl methyl-(2,2,2-trifluoroethyl) phosphonoacetate (5) and methyl dimethylphosphonoacetate (6). To a solution of methyl bis-(2,2,2-trifluoroethyl)phosphonoacetate (1) (324 mg, $1.02 \mathrm{mmol}$ ) in $\mathrm{MeOH}(1 \mathrm{~mL})$ was added aqueous $1 \mathrm{M} \mathrm{NaOH}(1 \mathrm{~mL})$ at $0{ }^{\circ} \mathrm{C}$. After being stirred at $0{ }^{\circ} \mathrm{C}$ for $45 \mathrm{~min}$, the reaction mixture was treated with $5 \% \mathrm{HCl}$ and then extracted with AcOEt $(20 \mathrm{~mL}$ $\mathrm{x} 5$ ). The extract was submitted to the usual workup to give an oily residue, which was purified by column chromatography on silica gel (Kanto Chemical N60) [ $n$-hexane/AcOEt (1:4) to $\left.\mathrm{CHCl}_{3} / \mathrm{MeOH}(20: 1)\right]$ to afford 5 (130 mg, 51\%) and 6 (36 mg, 20\%). 5; Colorless oil; ${ }^{1} \mathrm{H}$ NMR $\left(400 \mathrm{MHz}, \mathrm{CDCl}_{3}\right) \delta 3.07\left(2 \mathrm{H}, \mathrm{d},{ }^{2} J_{\mathrm{H}, \mathrm{P}}=21.5 \mathrm{~Hz}\right), 3.77(3 \mathrm{H}, \mathrm{s}), 3.84\left(3 \mathrm{H}, \mathrm{d},{ }^{3} J_{\mathrm{H}, \mathrm{P}}=11.7 \mathrm{~Hz}\right)$, $4.43\left(1 \mathrm{H}, \mathrm{d}\right.$ quint, $\left.{ }^{2} J_{\mathrm{H}, \mathrm{H}}=12.2 \mathrm{~Hz},{ }^{3} J_{\mathrm{H}, \mathrm{F}}={ }^{3} J_{\mathrm{H}, \mathrm{P}}=8.3 \mathrm{~Hz}\right), 4.51\left(1 \mathrm{H}\right.$, d quint, ${ }^{2} J_{\mathrm{H}, \mathrm{H}}=12.2 \mathrm{~Hz}$, $\left.{ }^{3} J_{\mathrm{H}, \mathrm{F}}={ }^{3} J_{\mathrm{H}, \mathrm{P}}=8.3 \mathrm{~Hz}\right) ;{ }^{13} \mathrm{C} \mathrm{NMR}\left(75 \mathrm{MHz}, \mathrm{CDCl}_{3}\right) \delta 33.6\left(\mathrm{~d},{ }^{1} J_{\mathrm{C}, \mathrm{P}}=140.8 \mathrm{~Hz}\right), 52.9(\mathrm{~s}), 53.1(\mathrm{~d}$, $\left.{ }^{2} J_{\mathrm{C}, \mathrm{P}}=6.9 \mathrm{~Hz}\right), 62.9\left(\mathrm{qd},{ }^{2} J_{\mathrm{C}, \mathrm{F}}=37.7 \mathrm{~Hz},{ }^{2} J_{\mathrm{C}, \mathrm{P}}=5.3 \mathrm{~Hz}\right), 122.8\left(\mathrm{qd},{ }^{1} J_{\mathrm{C}, \mathrm{F}}=277.4 \mathrm{~Hz},{ }^{3} J_{\mathrm{C}, \mathrm{P}}=8.1\right.$ $\mathrm{Hz}$ ), 165.7 (d, ${ }^{2} J_{\mathrm{C}, \mathrm{P}}=5.0 \mathrm{~Hz}$ ); IR (neat) 2964, 1743, 1264, 1174, 1094, $1041 \mathrm{~cm}^{-1}$; EI-MS calcd for $\mathrm{C}_{6} \mathrm{H}_{10} \mathrm{O}_{5} \mathrm{~F}_{3} \mathrm{P}$ MW 250.0218, found $\mathrm{m} / \mathrm{e} 250.0216\left(\mathrm{M}^{+}\right)$; Anal. calcd for $\mathrm{C}_{6} \mathrm{H}_{10} \mathrm{O}_{5} \mathrm{~F}_{3} \mathrm{P}$ : C, 28.81; $\mathrm{H}$, 4.03. Found: C, 28.85; H, 3.98\%. 6; ${ }^{9}$ Colorless oil; ${ }^{1} \mathrm{H} \mathrm{NMR}\left(400 \mathrm{MHz}, \mathrm{CDCl}_{3}\right) \delta 3.00(2 \mathrm{H}$, $\left.\mathrm{d},{ }^{2} J_{\mathrm{H}, \mathrm{P}}=21.7 \mathrm{~Hz}\right), 3.76(3 \mathrm{H}, \mathrm{s}), 3.82\left(6 \mathrm{H}, \mathrm{d},{ }^{3} J_{\mathrm{H}, \mathrm{P}}=11.2 \mathrm{~Hz}\right) ;{ }^{13} \mathrm{C} \mathrm{NMR}\left(75 \mathrm{MHz}, \mathrm{CDCl}_{3}\right) \delta$ $33.2\left(\mathrm{~d},{ }^{1} J_{\mathrm{C}, \mathrm{P}}=135.1 \mathrm{~Hz}\right), 52.7(\mathrm{~s}), 53.2\left(\mathrm{~d},{ }^{2} J_{\mathrm{C}, \mathrm{P}}=6.2 \mathrm{~Hz}\right), 166.1\left(\mathrm{~d},{ }^{2} J_{\mathrm{C}, \mathrm{P}}=6.2 \mathrm{~Hz}\right)$. 
Typical procedure for the HWE reaction of bis-(2,2,2-trifluoroethyl)phosphonoacetic acid (4) with i-PrMgBr

A $0.76 M$ solution of $i$-PrMgBr $(1.96 \mathrm{~mL}, 1.49 \mathrm{mmol})$ in THF was added to a stirred solution of bis-(2,2,2-trifluoroethyl)phosphonoacetic acid (4) (218 mg, $0.72 \mathrm{mmol}$ ) in anhydrous toluene (10 $\mathrm{mL}$ ) at $0{ }^{\circ} \mathrm{C}$ under argon. The mixture was stirred at $0^{\circ} \mathrm{C}$ for $1 \mathrm{~h}$ and then heated at reflux. 3Phenylpropionaldehyde (7a) (80 $\mu \mathrm{L}, 0.60 \mathrm{mmol}$ ) was added to the refluxing solution. After being heated at reflux for $1 \mathrm{~h}$, the reaction mixture was treated with $5 \% \mathrm{HCl}$ and then extracted with AcOEt (70 mL x3). The extract was submitted to the usual workup to afford a crude product 8a $(E: Z=95: 5)$. To the solution of $8 \mathbf{a}$ in $\mathrm{MeOH}(2 \mathrm{~mL})$ and benzene $(7 \mathrm{~mL})$ was added an excess amount of TMSCHN $_{2}(2.0 M$ solution in $n$-hexane, ca. $1 \mathrm{~mL}, c a .2 \mathrm{mmol})$. After being stirred at room temperature for $30 \mathrm{~min}$, the reaction mixture was evaporated in vacuo to afford a crude product, which was purified by chromatography on a silica gel (Kanto Chemical N60) column [n-hexane/AcOEt/acetone (50:2:1)] to afford (E)-9a (89 mg, 78\%) and (Z)-9a (4.7 mg, 4\%) as colorless oils.

Methyl (E)-5-phenyl-2-pentenoate [(E)-9a]. ${ }^{2 b, 10}$ Colorless oil; ${ }^{1} \mathrm{H}$ NMR (400 MHz, $\left.\mathrm{CDCl}_{3}\right) \delta$ 2.48-2.57 (2H, m), 2.77 (2H, t, $J=7.7 \mathrm{~Hz}), 3.72$ (3H, s), 5.85 (1H, td, $J=1.5,15.6 \mathrm{~Hz}), 7.01$ $(1 \mathrm{H}, \mathrm{td}, J=6.8,15.6 \mathrm{~Hz}), 7.16-7.24(3 \mathrm{H}, \mathrm{m}), 7.24-7.32(2 \mathrm{H}, \mathrm{m}) ;{ }^{13} \mathrm{C} \mathrm{NMR}\left(75 \mathrm{MHz}, \mathrm{CDCl}_{3}\right) \delta$ 33.9, 34.3, 51.4, 121.4, 126.2, 128.3, 128.5, 140.7, 148.4, 167.0.

Methyl (Z)-5-phenyl-2-pentenoate [(Z)-9a]. ${ }^{2 b, 10}$ Colorless oil; ${ }^{1} \mathrm{H}$ NMR (400 MHz, $\left.\mathrm{CDCl}_{3}\right) \delta$ $2.77(2 \mathrm{H}, \mathrm{t}, J=7.6 \mathrm{~Hz}), 2.96-3.03(2 \mathrm{H}, \mathrm{m}), 3.70(3 \mathrm{H}, \mathrm{s}), 5.79(1 \mathrm{H}, \mathrm{td}, J=1.5,11.5 \mathrm{~Hz}), 6.25$ $(1 \mathrm{H}, \mathrm{td}, J=7.4,11.5 \mathrm{~Hz}), 7.16-7.32(5 \mathrm{H}, \mathrm{m}) ;{ }^{13} \mathrm{C} \mathrm{NMR}\left(75 \mathrm{MHz}, \mathrm{CDCl}_{3}\right) \delta 30.5,35.0,51.1$, 119.9, 126.0, 128.4, 128.5, 141.1, 149.4, 166.7.

Methyl (E)-3-phenyl-2-propenoate [(E)-9b]. ${ }^{2 b, 11}$ Colorless plates (n-hexane), mp $32.5-33{ }^{\circ} \mathrm{C}$ (lit. $\left.{ }^{10 \mathrm{a}} 34-34.5{ }^{\circ} \mathrm{C}\right) ;{ }^{1} \mathrm{H}$ NMR (400 MHz, $\mathrm{CDCl}_{3}$ ) $\delta 3.81$ (3H, s), 6.44 (1H, d, $J=16.0 \mathrm{~Hz}$ ), 7.367.42 (3H, m), 7.50-7.56 (2H, m), $7.70(1 \mathrm{H}, \mathrm{d}, J=16.0 \mathrm{~Hz}) ;{ }^{13} \mathrm{C}$ NMR $\left(75 \mathrm{MHz}, \mathrm{CDCl}_{3}\right) \delta 51.7$, 117.8, 128.1, 128.9 130.3, 134.4, 144.9, 167.4.

Methyl (2E,4E)-5-phenyl-2,4-pentadienoate [(E)-9c]. ${ }^{12}$ White powder $\left(\mathrm{Et}_{2} \mathrm{O}-\boldsymbol{n}\right.$-hexane), mp $71{ }^{\circ} \mathrm{C}$ (lit. $\left.{ }^{11 \mathrm{~b}} 68-70{ }^{\circ} \mathrm{C}\right) ;{ }^{1} \mathrm{H} \mathrm{NMR}\left(400 \mathrm{MHz} \mathrm{CDCl}_{3}\right) \delta 3.78(3 \mathrm{H}, \mathrm{s}), 6.00(1 \mathrm{H}, \mathrm{d}, J=15.4 \mathrm{~Hz})$, 6.83-6.95 (2H, m), 7.28-7.40 (3H, m), 7.42-7.50 (3H, m); $\left.{ }^{13} \mathrm{C} \mathrm{NMR} \mathrm{(75} \mathrm{MHz,} \mathrm{CDCl}_{3}\right) \delta 51.6$, 120.8, 126.2, 127.2, 128.8, 129.1, 136.0, 140.5, 144.8, 167.5 .

Methyl (2Z,4E)-5-phenyl-2,4-pentadienoate [(Z)-9c]. ${ }^{12 a}$ Colorless oil; ${ }^{1} \mathrm{H}$ NMR (400 MHz, $\left.\mathrm{CDCl}_{3}\right) \delta 3.77(3 \mathrm{H}, \mathrm{s}), 5.74(1 \mathrm{H}, \mathrm{d}, J=11.0 \mathrm{~Hz}), 6.71-6.80(1 \mathrm{H}, \mathrm{m}), 6.83(1 \mathrm{H}, \mathrm{d}, J=15.6 \mathrm{~Hz})$, 7.27-7.39 (3H, m), 7.50-7.56 (2H, m), 8.14 (1H, dd, $J=11.5,15.6 \mathrm{~Hz}) ;{ }^{13} \mathrm{C} \mathrm{NMR}(75 \mathrm{MHz}$, $\left.\mathrm{CDCl}_{3}\right) \delta 51.2,116.9,124.8,127.5,128.7,129.0,136.3,141.4,145.0,167.0$.

Methyl (E)-3-cyclohexyl-2-propenoate [(E)-9d]. ${ }^{13 a, b}$ Colorless oil; ${ }^{1} \mathrm{H}$ NMR (400 MHz, $\left.\mathrm{CDCl}_{3}\right) \delta$ 1.07-1.38 (5H, m), 1.62-1.82 (5H, m), 2.08-2.20 (1H, m), $3.73(3 \mathrm{H}, \mathrm{s}), 5.77$ (1H, dd, $J=1.3,15.9 \mathrm{~Hz}), 6.92(1 \mathrm{H}, \mathrm{dd}, J=6.8,15.9 \mathrm{~Hz}) ;{ }^{13} \mathrm{C} \mathrm{NMR}\left(75 \mathrm{MHz}, \mathrm{CDCl}_{3}\right) \delta 25.7,25.9,31.7$, 40.4, 51.4, 118.5, 154.7, 167.6.

Methyl (Z)-3-cyclohexyl-2-propenoate [(Z)-9d]. ${ }^{13 c}$ Colorless oil; ${ }^{1} \mathrm{H}$ NMR (400 MHz, $\left.\mathrm{CDCl}_{3}\right)$

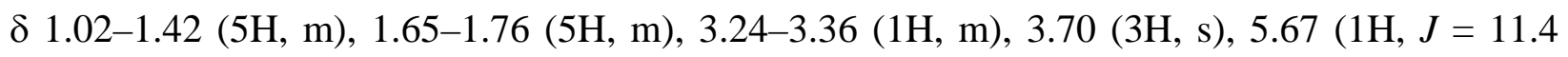


Hz), 6.04 (1H, dd, $J=10.1,11.4 \mathrm{~Hz}) ;{ }^{13} \mathrm{C} \mathrm{NMR}\left(75 \mathrm{MHz}, \mathrm{CDCl}_{3}\right) \delta$ 25.5, 25.9, 32.3, 37.3, 51.0, $117.1,156.1,166.8$.

\section{Acknowledgments}

This work was partially supported by a Grant-in-Aid for Scientific Research (C) from the Japan Society for the Promotion of Science.

\section{References}

1. (a) Maryanoff, B. E.; Reitz, A. B. Chem. Rev. 1989, 89, 863. (b) Rein, T.; Reiser, O. Acta Chem. Scand. 1996, 50, 369. (c) Sano, S. Yakugaku Zasshi 2000, 120, 432. (d) Rein, T.; Pedersen, T. M. Synthesis 2002, 579.

2. (a) Still, W. C.; Gennari, C. Tetrahedron Lett. 1983, 24, 4405. (b) Sano, S.; Yokoyama, K.; Fukushima, M.; Yagi, T., Nagao, Y. Chem. Commun. 1997, 559. (c) Sano, S.; Takehisa, T.; Ogawa, S.; Yokoyama, K.; Nagao, Y. Chem. Pharm. Bull. 2002, 50, 1300.

3. (a) Machleidt, H.; Wessendorf, R. Liebigs Ann. Chem. 1996, 674, 1. (b) Burton, D. J.; Yang, Z. -Y.; Qin, W. Chem. Rev. 1996, 96, 1641. (c) Sano, S.; Ando, T.; Yokoyama, K.; Nagao, Y. Synlett 1989, 777. (d) Sano, S.; Yokoyama, K.; Shiro, M.; Nagao, Y. Chem. Pharm. Bull. 2002, 50, 706.

4. (a) Sano, S.; Teranishi, R.; Nagao, Y. Tetrahedron Lett. 2002, 43, 9183. (b) Sano, S.; Saito, K.; Nagao, Y. Tetrahedron Lett. 2003, 44, 3987.

5. Supporting information available, see: Ghosh, A. K. J. Org. Chem. 2001, 66, 8973.

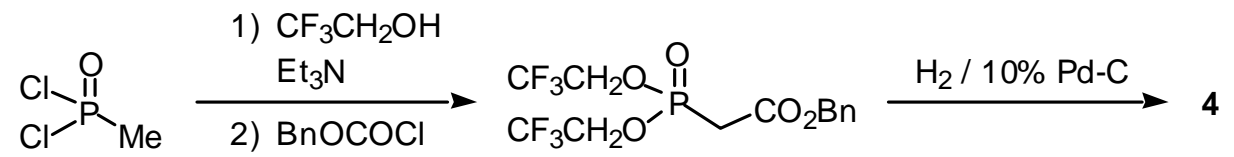

6. Shioiri, T.; Aoyama, T. J. Synth. Org. Chem. Jpn. 1986, 44, 149.

7. Shen, Y.; Wang, G.; Sun, J. J. Chem. Soc., Perkin Trans. $12001,519$.

8. (a) Sano, S.; Kobayashi, Y.; Kondo, T.; Takebayashi, M.; Maruyama, S.; Fujita, T.; Nagao, Y. Tetrahedron Lett. 1995, 36, 2097. (b) Sano, S.; Liu, X-K.; Takebayashi, M.; Kobayashi, Y.; Tabata, K.; Shiro, M.; Nagao, Y. Tetrahedron Lett. 1995, 36, 4101. (c) Hayashi K.; Kogiso, H.; Sano, S.; Nagao, Y. Synlett 1996, 1203. (d) Sano, S.; Miwa, T.; Liu, X.-K.; Ishii T.; Takehisa, T.; Shiro, M.; Nagao, Y. Tetrahedron: Asymmetry 1998, 9, 3615. (e) Sano, S.; Ishii, T.; Miwa, T.; Nagao, Y. Tetrahedron Lett. 1999, 40, 3013. (f) Sano, S.; Nagao, Y. J. Synth. Org. Chem. Jpn. 2000, 58, 756.

9. Fieser, M.; Fieser, L. In Reagents for Organic Synthesis; John Wiley Sons: New York, 1969; Vol. 2, p 442.

10. Hon, Y.-S.; Lee, C.-F. Tetrahedron 2000, 56, 7893. 
11. (a) Kunishima, M.; Kawachi, C.; Morita, J.; Terao, K.; Iwasaki, F.; Tani, S. Tetrahedron 1999, 56, 13159. (b) Keck, G. E.; McLaws, M. D.; Wager, T. T. Tetrahedron 2000, 56, 9875.

12. (a) Nakamura, S.; Hayakawa, T.; Nishi, T.; Watanabe, Y.; Toru, T. Tetrahedron 2001, 57, 6703. (b) O’Donnell, M. E.; Sanvoisin, J.; Gani, D. J. Chem. Soc., Perkin Trans. 1 2001, 1696.

13. (a) Huang, Y.-Z.: Shi, L.-L.; Li, S.-W.; Wen, X.-Q. J. Chem. Soc., Perkin Trans. 1 1989, 2397. (b) Zhou, Z.-L.; Huang, Y.-Z.; Shi, L.-L. Tetrahedron 1993, 49, 6821. (c) Ando, K. J. Org. Chem. 1999, 64, 8406. 